\title{
UNIVERSAL ETHICS: ORGANIZED COMPLEXITY AS AN INTRINSIC VALUE
}

\author{
Clément Vidal \\ Center Leo Apostel \& \\ Evolution Complexity and Cognition, \\ Vrije Universiteit Brussel, \\ contact@clemvidal.com
}

\author{
Jean-Paul Delahaye \\ Centre de Recherche en Informatique, \\ Signal et Automatique de Lille (CRISTAL) \\ Université de Lille 1 \\ jean-paul.delahaye@univ-lille1.fr
}

\begin{abstract}
How can we think about a universal ethics that could be adopted by any intelligent being, including the rising population of cyborgs, intelligent machines, intelligent algorithms or even potential extraterrestrial life? We generally give value to complex structures, to objects resulting from a long work, to systems with many elements and with many links finely adjusted. These include living beings, books, works of art or scientific theories. Intuitively, we want to keep, multiply, and share such structures, as well as prevent their destruction. Such objects have value not because more information (in bits) would simply mean more value. Instead, they have value because they require a long computational history, numerous interactions for their construction that we can assimilate to a computation, and they display what we call organized complexity. To propose the foundations of a universal ethics based on the intrinsic value of organized complexity, we first discuss conceptions of complexity, and argue that Charles Bennett's logical depth is certainly a first approximation of what we are looking for. We then put forward three fundamental imperatives: to preserve, augment and recursively promote organized complexity. We show a broad range of applications with human, non-human and non-living examples. Finally, we discuss some specific issues of our framework such as the distribution of complexity, of managing copies and erasures, and how our universal ethics tackles classical ethical issues. In sum, we propose a clear, homogenous and consistent ethical foundation that can integrate many universal ethics desiderata.
\end{abstract}

KEYWORDS: Philosophy of information, organized complexity, Kolmogorov complexity, logical depth, ethics, universal ethics, infoethics, thermoethics, environmental ethics, transhumanism

1. Introduction

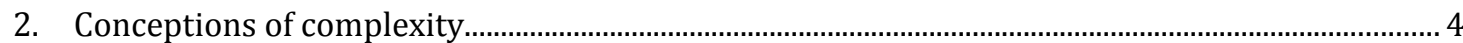

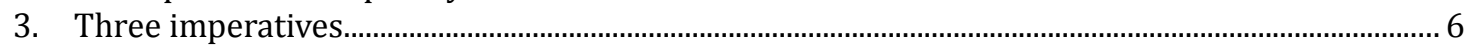

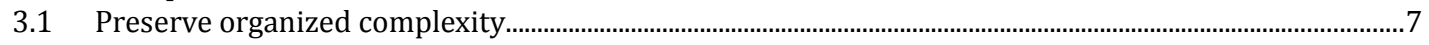

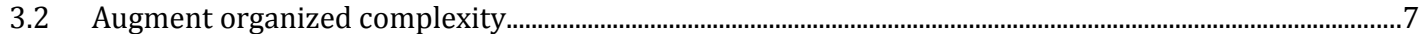

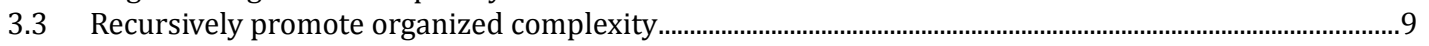

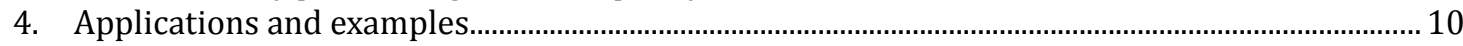

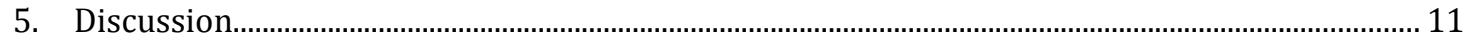

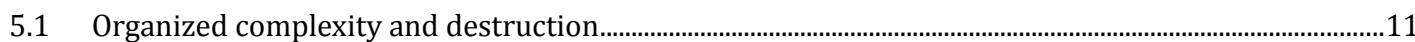

5.2 Organized complexity and traditional ethical issues...............................................................................................12

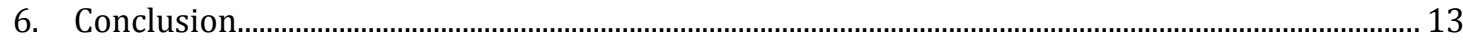

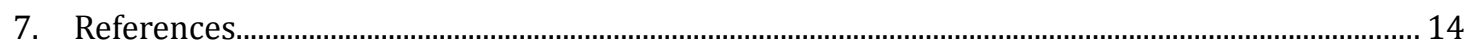

\section{Introduction}

Humans have developed multiple kinds of ethical systems, in different parts of the world, based on religious or humanist values. In our global and postmodern era, moral relativism makes a strong case, but by definition, it is not attempting to find a foundation for ethics.

Most of the time, ethical systems are anthropocentric in the sense that they value human beings above anything else. This is embarrassing, as a wellfounded normative theory should also be able to answer questions not only about human values, but also other value-related questions, such as aesthetic ones, for example: "why does a symphony have value?". 
Furthermore, ethical systems from various cultures are often incompatible between each others, generating conflicts and violence at all scales: communal, societal, national and international.

In this paper, we propose a new concept for the foundation of a universal ethics. We mean universal, both in a physical, cosmological sense, as well as in an abstract and mathematical sense. The "universal" declaration of human rights is not universal in this sense, as "universal" in the declaration refers simply to all human beings. It is unlikely that this declaration would make any sense for an extraterrestrial being in another galaxy.

Two kinds of universal ethical systems have been proposed. The first kind is based on matter-energy processes.

For example, thermoethics' central principle is to make the most of free energy, and to avoid the production of unnecessary waste, disorder and entropy (it is also called "entropy ethics", see Ostwald 1912; Freitas Jr 1979, sec. 25.1.3; Hammond 2005; Korbitz 2010; Vidal 2014, chap. 10).

Another example of a path towards a universal ethics could be based on the concept of emergy (with an " $m$ "). The concept comes from systems ecology, and measures an energetic content. It is defined as the value of a system, be it living, social or technological, as measured by the solar energy that was used to make it (e.g. Odum 2007).

Although underexplored, the focus of our paper will not be on the possibility of a universal ethics based on matter-energy. Instead, we will focus on the second kind of universal ethics, based on information and computation. With the rise of the information society, and the importance and ubiquity of computers in our world, information exchanges have become predominantly relevant and this is why a philosophy based on information and computation is needed (for an introduction to the field, see Floridi 2003).

An information and communication approach to universal ethics can be based on cybernetics, as it is a general science of control and communication (for some steps in this direction, see e.g. Beer 1997; Chambers 2001; Vidal 2014, 285-86). Focusing on information science, Floridi (2008) did develop the concept of infoethics, that bears similarities with thermoethics (Vidal 2014, 271). To deepen and broaden traditional ethical systems, Ward Bynum (2006) did initiate the seeds of a universal ethics based on information that can apply to every physical entity in the universe. However, the concept of information used in this approach is mostly semantic, and has been criticized for its vagueness (e.g. Adriaans 2010). In this paper, we introduce and focus on a syntactic computational concept of valuable information to found a universal ethics. This concept is logical depth (Bennett 1988). We think that this approach solves the issues that have been raised about infoethics: the basic notion is purely syntactic and mathematical, without the need to refer to cognitive agents such as humans. By contrast, the concept of semantic information that would be "well-formed, meaningful, and truthful data" is so delicate to define and leads to so many difficulties that it would risk to make vague any ethical system based on it.

What are the desiderata of a universal ethics? A universal ethics should first be able to justify existing invariant values in humans, and provide a better understanding in the existence of such commonalities. As a matter of fact, there are broad invariants in human ethical systems: murder, theft, rape, lying, and more generally destruction are negative values in all societies, whereas health, wealth, friendship, honesty, safety, freedom, and equality are positive ones (e.g. Heylighen and Bernheim 2000). 
A universal ethics should also be able to give value both to humans and their cultural products. These include works of art, scientific theories, books or libraries. We intuitively know that burning libraries of museums is something "bad".

A universal ethics is also expected to support the widening of moral boundaries (see Fig. 1). We rarely see ethical concerns going beyond humanity. Fortunately, this is changing as consciousness is rising to care and value the nonliving Earth's climate, realizing that taking care of it is necessary for our longterm survival. Some religions such as Buddhism also do care about "all beings", which may include all life on Earth and in the Universe.

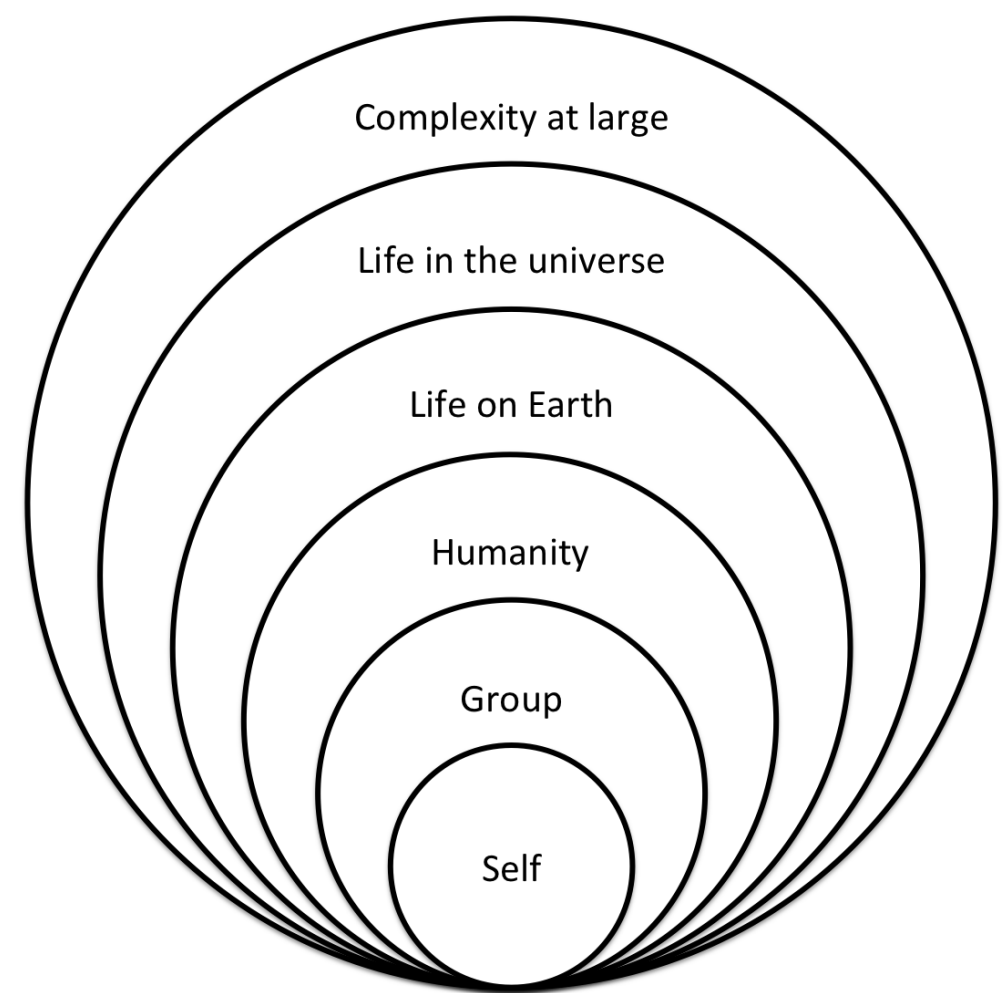

Figure 1 - The widening of moral boundaries. Historically, humans have cared for increasingly wider systems. The self gradually cares for the group, that extends from a family, a community, a nation to the whole of humanity. We extend the last circles of compassion to the notion of complexity at large, not limited to living things.

Such widening of moral boundaries has taken several shapes, from the rise of animal rights (Bentham 1907, chap. XVII; Regan 1987), to taking care of all living things on Earth, for example with biocentric ethics (Agar 2001), deep ecology (Næss 2008), or environmental ethics. Indeed, the ecological worldview according to which all living things and the Earth are connected requires caring about non-human living processes that support the living realm.

In our digital era at the dawn of transhumanism, humans are transforming and re-shaping themselves through genetic engineering and technology. We are seeing the rise of the first augmented humans (or cyborgs) and this raises entirely new moral issues that are hard to deal with traditional ethical systems. We thus need insights and frameworks to develop a transhumanist ethics, to deal with relations between humans and transhumans, as well as their respective relations with machines. A related field is machine ethics, (e.g. Anderson and Anderson 2011) that deals with moral aspects of machine-machine interactions. 
Finally, a genuinely universal ethics should lead consistently to an extraterrestrial ethics, i.e. an ethics that can meaningfully apply to potential extraterrestrial life (see Vakoch 2014). This field explores issues regarding our obligations and duties towards a new lifeform we may discover (see e.g. issues raised by planetary protection programs in Meltzer 2010). As a thought experiment, it is also challenging and mind broadening to think about the kinds of ethical behaviors and principles we can expect from potential extraterrestrial intelligence (see e.g. Ruse 1985), as a way to prepare for the impact of the discovery of extraterrestrial life (Vidal 2015).

In this paper, we first discuss various conceptions of complexity, and introduce the notion of organized complexity, based on the computational concept of logical depth. Then we show how organized complexity can be put to use as an intrinsic value, leading to three core imperatives: that we should preserve and augment existing organized complexity, as well as recursively promote systems that increase organized complexity. We illustrate our framework with some applications and examples. Finally, we discuss various issues that arise from this original computational approach to universal ethics.

\section{Conceptions of complexity}

There is no doubt that multicellular organisms are more complex than unicellular ones. On large time scales, it is generally agreed that there is a growth in complexity of living beings that appear in evolution (e.g. Coren 1998; Livio 2000; Mayfield 2013; Delahaye and Vidal 2018). Both biological and technological evolution produce more and more complex objects, that is, displaying richer and richer structures.

A precise definition of such " complexity » is hard to come with, and to formulate mathematically (for a review, see Bennett 1990). Many ideas have been attempted, although they are often inadequate. For example, Eric Chaisson (2001) defines complexity as the rate of energy flowing through a system, normalized by its mass. This idea of energy rate density is at first sight seducing, as it can apply to many epochs of cosmic evolution, from galaxy formation, planet formation, to living systems and our technological society.

However, it has the drawback that it is totally blind to the concepts of computation or information. There is no stable correspondence between energy and computation. As a matter of fact, a variant of Moore's law shows that since the 1960s the number of computation per unit energy is doubling every year and a half (Koomey et al. 2011). A modern microprocessor is clearly more complex than a computer made 40 years ago, in the sense that for a given amount of energy, it can perform one million more operations. Structurally, a modern microprocessor also contains one million more transistors. All this complexity features are not captured by a metric based on energy only.

Complexity can also rise in artificial life simulations, such as J. H. Conway's game of life (Berlekamp, Conway, and Guy 2001) where energy plays no role. Another example is the complexity of a musical piece. It is not the sound's loudness, its frequencies, nor its tempo that seems to determine its complexity, but its mathematical structure, without the need of any thermodynamic notion. Yet another example is a painting or a sculpture. According to Chaisson's metric, since no energy flows through them, they have zero complexity, and zero value if we would take complexity as a guide for axiology. 
Although useful to study ecosystem complexity (Ulgiati and Brown 2009), one can note that the concept of emergy is not a good general proxy for complexity. For example, a lot of energy is necessary to produce an ingot of aluminium, but this doesn't mean that an ingot of aluminium is particularly complex. It thus seems valid to search for a concept of complexity that is not founded on energy and its circulation, that is, a non-thermodynamic concept of complexity.

In theoretical computer science, two main notions of complexity have been proposed: Kolmogorov complexity and Bennett's logical depth (Li and Vitányi 2008; Bennett 1988; Delahaye 2009). These notions concern finite numerical objects, that are assimilated - without loss of generality - to finite strings $s$ of ' 0 ' and ' 1 '. The Kolmogorov complexity of $s$ is by definition the size of the shortest program $s^{*}$ that outputs the string $s$ (for example, by printing it or by writing it in an output file). This is a useful notion in many respects, but it is not suitable as a measure of complexity if we consider complexity to be strongly structured. The notion does not cover the idea of complexification that scientists use when discussing biological or technological evolution. Indeed, strings that have the highest Kolmogorov complexity are random strings, and we intuitively know that randomness is the opposite of organized complexity.

Fortunately, based on Kolmogorov complexity, the notion of logical depth seems to be suitable as a definition of organized complexity, at least in a first approximation. The logical depth of $s$ is by definition the computation time that it takes for the program $s^{*}$ to produce $s$. In the case of an object $s$ with a low complexity (e.g. a repetitive string like $000000 \ldots 00$, or a random string) this computation time is minimal; while as soon as $s$ is a complex and structured object, the computation time of $\mathrm{s}^{*}$ to produce $s$ increases.

For example, the string of the first million digits of Pi has a large logical depth, as well as the string of a musical piece translated into 0 and 1 , or the string of prime numbers. The logical depth can either be seen as a measure of the quantity of structures in $s$, or as a quantity of computation present in $s$.

This definition of logical depth thus embeds two core values. First, because it is based on Kolmogorov complexity, it values the effort to find short, shorter or the shortest programs. This is the fundamental epistemic value of searching the simplest and shortest models in science. Given some data to explain, if one finds a new simpler, shorter theory, it is to be preferred to a longer one. This is known by philosophers of science as Occam's razor, but many computer scientists have formalized it using Kolmogorov complexity, showing the links between machine learning and compression (e.g. Blumer et al. 1987; Li, Tromp, and Vitányi 2003; Li and Vitányi 1992; 2008; Delahaye 1994).

The second value is that the longer it took for an object to appear (assuming that it can not be obtained in a simpler way), the more it has value. This value is specific to the definition of logical depth, and the consequences of which we develop in this paper.

Some aspects of normative evolutionary ethics valuing complexity growth and diversity are compatible with the ethics of organized complexity. For example, the survival instinct is clearly protecting the living complexity of the organism, while the reproductive instinct secures the preservation of genetic information through generations. Mutations, sexual reproduction, coupled with selection from the environment lead to a complexification of organisms on large time scales. How and in how far we should be inspired by evolutionary processes 
to build a normative ethics remains a huge debate worth pursuing (see e.g. Maienschein and Ruse 1999; Quintelier, Speybroeck, and Braeckman 2011).

Evolution produces complex organisms by operating many and diverse choices. Such elementary operations constitute what Dennett (1995) calls a "Darwinian algorithm », that we can assimilate to the creation of computational contents stored in living beings (see also Mayfield 2013). This algorithmic view can be naturally extended to cosmic evolution (Delahaye and Vidal 2018).

Complex organisms can only appear when less complex ones have already appeared. This corresponds to a slow growth law, that has been demonstrated for Bennett's logical depth. It shows that complexity cannot appear suddenly, that it requires a long time of maturation. This can be illustrated with an example from the history of science, namely, the now refuted idea of spontaneous generation (Strick 2000). From this computational perspective, spontaneous generation could have been a priori refuted, as it would be extremely improbable that sophisticated, complex living systems would appear in a few days.

An ethical foundation based on this definition of complexity is thus conceivable. The good becomes what contributes to the preservation and augmentation of structural contents, in non-living, living beings, humans, and their cultural products, whether they are artistic, scientific, technic, economical or political. Such an ethics suggests a respect and a protection not only of human and living beings, but also of all objects and structures that accompany them, or that further organizes them. Rich structures have required large computational work and this is why we must protect them.

A work of art (a painting, a symphony or a novel) is an object with a high content in computation, as it is the result of a long elaboration. Its organization is rich and not trivial: it is an object with a great logical depth i.e. computational content, that must be respected, protected because it has value in such an ethics of organized complexity. The same holds for a science book, a microprocessor, the form of organization of our societies, the networks of interconnections of our cities and countries, etc.

It is remarkable that some cryptocurrencies such as Bitcoin or Ethereum have at their core a record (called the blockchain) that has a strong content in computation, i.e. a great logical depth. This makes them nearly unfalsifiable in practice, and constitutes a concrete example linking in practice the concept of logical depth with value.

One may note that other ethics may be developed and supported with other existing or future conceptions of complexity, leading to somewhat different results, and this may be a worthwhile effort. However, it seems to us that the notion of logical depth lends itself naturally to the project of a nonthermodynamic universal ethics, as it offers a robust, adequate and precise formal definition. We want to show that it encompasses what we naturally consider to have value.

\section{Three imperatives}

We identify three imperatives of the ethics of organized complexity: to preserve, augment, and recursively promote what preserves and augments organized complexity. As these are fundamental imperatives, more ethical consequences may be derived from them, an exercise which we will not attempt to do systematically in this paper. 


\subsection{Preserve organized complexity}

The first imperative is to preserve existing organized complexity. Indeed, organized complexity took time and effort to appear, so it intuitively makes sense to preserve and protect it. We have inbuilt biological survival instincts that lead us to preserve ourselves and our offsprings. Humans also have a tendency to systematically collect and store organized complexity. For example, we put efforts to preserve biodiversity, or to protect endangered species and also everything that has needed an effort to be obtained: a painting, a monument, a patented idea. We are aware of the value of our cultural heritage, that we should avoid the destruction of rare buildings, objects, or works of art. Historical and cultural preservation organizations exist worldwide, to preserve all kinds of complex human structures (consider for example UNESCO's world heritage sites, or many other national heritage protection programs).

In the digital world, this preservation imperative implies to have serious backup strategies of our data. Most of us have experienced data loss and know how bad this can be.

Even if the complexity collected has no immediate pragmatic value, it may have value in the future. This is true for biological evolution, where noncoding DNA sequences are conserved in the genome, which may in future generations be actived. This is also true in mathematics, where theories that once had no practical use are nowadays central tools for science and technology. Classical examples include the use of non-euclidian geometries for relativity theory or the use of arithmetic for modern cryptography. A similar dynamic is likely to apply in the future, which urges us to carefully preserve and make accessible theoretical scientific knowledge.

If systematic collection and storage is not possible, we need to think about heuristics regarding what to collect, what to store, and what to make most accessible. In computer science, this is related to the recurrent problem of managing memory (space) and speed (time) and leads to space-time tradeoffs.

One may object that losing old structural products of complexity is not so grave, as long as we are able to preserve their function. For example, even if Galileo's original telescope is not constructed anymore, we have much more powerful and reliable telescopes today, so the loss is limited. Another example is the computer. Nobody misses vacuum tube computers, what is important is that we have general purpose computers that can do the same operations than these early computers. So, the preservation of organized complexity can be both structural and functional.

Also, as a first heuristic, one may suggest to adapt our preservation strategy relative to the number of copies. If there is 100 copies of a book, it is less grave to destroy one of them, than if we would destroy a unique copy of the same book.

\subsection{Augment organized complexity}

One can emphasize the preservation and conservation of the old, but creation of novelty, and augmentation of organized complexity is as valuable and important. We saw that the slow growth law (Bennett 1988) implies that we can't just quickly create deep objects out of nothing. We need time and to build on previous efforts, and this leads us to our second imperative: augment organized complexity. 
This raises the question: how? This is the issue of the distribution of organized complexity: should we try to augment the overall, total organized complexity, or the average organized complexity? The issue is similar to classical discussion of the total versus average utility issue in utilitarism (Sidgwick 1907). The issue is actually a particular instance of a more general problem of optimal allocation of resources, of defining social welfare functions (Chevaleyre et al. 2006).

To define the issue more precisely, let us imagine that we have a partition of the universe in well-defined components (humans, countries, celestial bodies, galaxies, etc.), that we note $C_{1}, C_{2}, \ldots, C_{n}$. There are different goals that one may want to pursue to preserve and augment organized complexity. For example :

Goal 1 : Augment organized complexity as a whole, as the union of: $\mathrm{C}_{1}+\mathrm{C}_{2}$ $+\ldots+C_{n}$.

Assuming that this organized complexity is measured adequately by the logical depth (LD), the goal is thus to maximize $\mathrm{LD}\left(\mathrm{C}_{1}+\mathrm{C}_{2}+\ldots+\mathrm{C}_{\mathrm{n}}\right)$ that we note as :

$$
\operatorname{maximize}\left(\mathrm{LD}\left(\mathrm{C}_{1}+\mathrm{C}_{2}+\ldots+\mathrm{C}_{\mathrm{n}}\right)\right)
$$

This may be called a global conception.

Goal 2: Augment the sum of organized complexity inside the different components :

maximize $\left(\mathrm{LD}\left(\mathrm{C}_{1}\right)+\mathrm{LD}\left(\mathrm{C}_{2}\right)+\ldots+\mathrm{LD}\left(\mathrm{C}_{\mathrm{n}}\right)\right)$.

This is not the same as Goal 1 , because if $\mathrm{C}_{1}$ and $\mathrm{C}_{2}$ are identical, we have :

$\mathrm{LD}\left(\mathrm{C}_{1}+\mathrm{C}_{2}\right)=\mathrm{LD}\left(\mathrm{C}_{1}\right)<\mathrm{LD}\left(\mathrm{C}_{1}\right)+\mathrm{LD}\left(\mathrm{C}_{2}\right)$

More generally, the more common structures exist between $\mathrm{C}_{1}$ and $\mathrm{C}_{2}$, the greater the difference is between $\operatorname{LD}\left(\mathrm{C}_{1}\right)+\operatorname{LD}\left(\mathrm{C}_{2}\right)$ and $\operatorname{LD}\left(\mathrm{C}_{1}+\mathrm{C}_{2}\right)$. In this context, we may call this an additive conception.

Goal 3 : Maximize the least structurally complex components : maximize (minimum $\left(\mathrm{LD}\left(\mathrm{C}_{1}\right), \mathrm{LD}\left(\mathrm{C}_{2}\right), \ldots, \mathrm{LD}\left(\mathrm{C}_{\mathrm{n}}\right)\right.$ ).

This is an egalitarian conception, aiming at making the poorest the least possible so.

Goal 4 : Maximize the organized complexity of the best components : maximize (maximum $\left(\mathrm{LD}\left(\mathrm{C}_{1}\right), \mathrm{LD}\left(\mathrm{C}_{2}\right), \ldots, \mathrm{LD}\left(\mathrm{C}_{\mathrm{n}}\right)\right.$ ).

In this case, it is an elitist conception : it doesn't matter if some components have a low organized complexity, what matters is to increase the organized complexity of the already highest complexity components.

Goal 5 : Maximize the product of organized complexity : maximize $\left(\mathrm{LD}\left(\mathrm{C}_{1}\right) \times \mathrm{LD}\left(\mathrm{C}_{2}\right) \times \ldots \times \mathrm{LD}\left(\mathrm{C}_{\mathrm{n}}\right)\right)$.

This is a compromise between goal 3 and goal 4 that avoids that certain components are too penalized. This conception is used in optimal affection of resources and is called Nashian (Ramezani and Endriss 2010). 
We will not discuss how one might settle these different goals and viewpoints, as each of them has arguments in its favor. What we want to argue is that the richness of a component should be measured by its organized complexity, and that it is a universal and coherent ethical position.

Let us note that as soon as a metric to measure the value of components in the world is given, in any valuation system, this allocation of resources issue rises, and we need to choose between a global, additive, egalitarian, elitist or Nashian conception. Even if the measure is not clear-cut or possible in practice, the different goals underly different philosophical, political and ethical choices.

It is also worth noting that informational resources are non-rival, and can be shared with a negligible cost compared to rival, matter-energy resources. So a different strategy and treatment might be necessary for the distribution of rival and non-rival resources. For example, it is natural and easy to be egalitarian and to share knowledge with all humans via the internet, whereas doing the same with oil is less obvious.

\subsection{Recursively promote organized complexity}

The third imperative is to recursively promote what preserves and augments organized complexity. In other words, it is to create, value and help systems and strategies that can preserve and augment $\mathrm{OC}$, to the $\mathrm{n}^{\text {th }}$ order.

Let us illustrate this with two examples: the mathematician and the musician. At the recursive level 0 , the mathematician finds a new theorem, and the composer composes a new piece of music. They can make efforts to diffuse their works for example by writing a book or recording a CD. This is a level 1 effort, as it limits the risk that the organized complexity created could brutally disappear. At level 2, the funding of a library, or a multimedia library will allow to preserve and diffuse organized complexity when the book or the CD will be released. Participating and helping a government whose goal is to collect taxes to build libraries is at level 3.

One can note that a library security system does not have a strong intrinsic complexity, but is still valuable to preserve existing OC, recursively. Jacques Monod $(1972,180)$ defended such a higher-order way to preserve knowledge, with his ethic of knowledge that "prescribes institutions dedicated to the defense, the extension, the enrichment of the transcendent kingdom of ideas, of knowledge, and of creation".

Generally, copies also help this recursive imperative. For example, libraries that store copies of books help to further build complexity, as they give access to existing deep work, on which further complexity can be built. In our digital era, it becomes obvious that all kinds of open source and open access initiatives are cheap and highly beneficial and should therefore be promoted (e.g. Heylighen 2007; Steele 2012).

There are indirect ways to promote organized complexity, namely to ensure that the supporting systems are effective. For example, we need to care about Earth's climate for the preservation and augmentation of biological complexity. Earth's climate is non-human, non-living, but it should still be taken care of. Another example is the requirement of energy to build organized complexity. Energy has value in the sense that it is a potential to build organized complexity. This ties in with the values of thermoethics. Unfortunately, these recursive and indirect ways to promote organized complexity make the assessment of value more complicated in practice. 


\section{Applications and examples}

If one adopts organized complexity as a universal value, then it is possible to naturally recover a large number of values accepted in many ethical theories. Let's see a few examples.

Every human being is a complex construct resulting from his genes, his education and the experiences he has had. This construction is unique and it has a logical depth which even if we do not know how to measure precisely, does not make the slightest doubt. To kill a human being is from the point of view of the ethics of organized complexity a bad action. In the same way, everything that degrades, disturbs, paralyzes, hurts a human being by making it less effective and simply by damaging the structural richness that it carries must be recognized as bad from this point of view.

The ethics of organized complexity commands us in the same way to respect and protect animals and, in a general way, the living. Interestingly enough, this ethics gives special importance to endangered species, as their individuals carry an organized complexity that will be impossible to recover if the species disappears. The difference of attention to be paid to a living being belonging to a species represented by millions of individuals and a living being existing only in a few copies is naturally taken into account by the ethics of organized complexity.

In the case of human beings, a strict application of organized complexity would at first sight lead to give more value to a genius than to the average person. However, we generally assume that all human lives have equal value. Indeed, to suppose the opposite would create social and political difficulties, so the solution admitted in democracy is that all humans have equal rights.

Another example of the immediate application of the ethics of organized complexity concerns works of art. They have an obvious structural content and the talent recognized by the artists who produce them is linked to their ability to elaborate (to calculate) in a new way complex, original structures, which is a form of logical depth. When the content in computation is not in the work itself seen as an isolated object, as in minimal art, it is in the new relations which it establishes between the world and the work of art that has value, relation that is also the creation by the artist of organized complexity.

In addition, the emotion provoked by a work of art stimulates the mind, even for minimalist works, because it surprises the brain. These emotions create a new mental state, creating new neural connections, inspiring us to think differently. A positive emotion enriches and therefore increases the wealth in the organization of the brain.

Generally speaking, we recognize that a work of art has value in proportion to its internal structural richness and its novelty. When it offers a new perspective on the world we give it value in proportion to the fineness and subtlety of what it shows us of the world, that we can interpret as a new structural wealth established by the work of art and the world. Anything that makes us recognize value in a work of art can easily be interpreted as structural richness, and therefore the idea that works of art must be preserved and protected is a direct application of the principles of the universal ethics of organized complexity.

Music is a particularly striking example of the purely structural content we perceive, and humans value many different kinds of music styles. While the formation of our ear will make us appreciate differently European or Indian 
music, an effort quickly leads us to love even music to which we have not been accustomed. In the end what we love and what makes a musical work worthwhile for us is its richness in structure.

Science, too, can fall within the value scheme proposed by the ethics of organized complexity. The most important scientific theories that would be morally condamnable to forbid, or not to diffuse are those which asked for a big work (experimental, conceptual, mathematical, etc.). Those big, deep works have a great content in computation or equivalently, in structure. Here again, our proposition of universal ethics brings us directly to these general values which are grounded under a single, homogeneous concept.

\section{Discussion}

\subsection{Organized complexity and destruction}

A delicate question that arises naturally if we adopts organized complexity as a value is the erasure of data or, equivalently, the destruction of structures. Destruction seems to be the direct opposite value of promoting organized complexity. Yet, at least three factors determine the "right" decision to make in concrete cases.

First, the existence or non-existence of other copies of the data or structure in question. If there are many copies, erasing a few redundant copies will not affect the global organized complexity. One may thus argue that their destruction is not (so)"bad".

However, as we saw, there is a second factor one may want include if one adopts a principle of egalitarianism for managing organized complexity. In this case, destroying complexity at one point could have the effect of lowering the organized complexity of the component of the universe considered, and would thus be "bad".

A third difficulty arises when taking into account our third imperative of recursively promoting organized complexity. This recursive promotion often requires to keep, at least temporarily, some data or structures. The problem is similar to that of information management in a computer system: the efficiency sometimes requires that the same data is copied several times, in order to have optimal access to it, and thus to compute the desired results more quickly. Here, even if immediately it is not useful to keep the intermediate data or structures in question because they do not contribute to final desired result directly, it is possible that keeping copies of such data guarantees a better creative potential and therefore the "good" choice is to destroy nothing.

Another aspect of this algorithmic issue is that some data are easy to reconstruct and thus keeping them clutters the space or memory the system. Good management then means to destroy such data. For example, modern algorithms for testing the primality of a number, or for the fast generation of prime numbers make it unnecessary to build and store large tables of prime numbers. However, it made sense to do so in a world without computers, and indeed, in the nineteenth century building such tables gave rise to works and publications. In this case, the technological progress in computing has tipped the right decision from "keep" to "delete".

In sum, even by adopting a mathematically well defined point of view for assessing value, the precise determination of actions recommended by an ethics of organized complexity does not lead in practice to simple solutions. On the contrary, it inherits all the difficulties necessary to conceive effective algorithms. 


\subsection{Organized complexity and traditional ethical issues}

Let us outline a few connections between our proposal and some traditional ethical issues.

We defended organized complexity as a foundational intrinsic value, thus apparently implying a moral monism. However, in an applied ethics context our framework is obviously not able to directly lead to solutions in a deterministic way. A pragmatic option is thus to incorporate this central value of organized complexity within existing ethical theories, thus opening the road to a moral pluralism. This combination with existing ethical theories may possibly enrich and deepen them (see also Bynum 2006).

It may be possible - and desirable - to consider the rise of organized complexity as the utility function in an utilitarian framework (instead of, classically, augmenting pleasures and reducing pains). Of course, it remains very hard to foresee what actions will preserve and augment complexity, especially if we consider our third principle of recursively promoting organized complexity.

However, this issue is not specific to our approach as any consequentialist must have good models of the world, to anticipate the future as well as possible. This leads to the cognitive value of modeling, i.e. the anticipation of our actions and the world. On a short time scale, we can all agree that having the reflex to avoid a falling rock is good, and the skills to climb in a tree to collect a delicious fruit is also good and adaptive. We need similar skills at higher spatial and temporal levels and be able to anticipate and model the future of groups, societies, Earth, the universe and complexity at large. In practice, a trade-off must often be found between the thinking and acting mindsets. Too much thinking and planning paralizes action, and too little thinking can lead to risky and premature actions.

Instead of taking organized complexity as an intrinsic normative value, one could also use it as a descriptive value, in the sense that it may help to explain why certain behaviors or objects are intuitively considered to have value by an agent. One may thus try to explain an existing moral valuation in terms of its preservation and augmentation of organized complexity.

We can recover the value of striving for happiness, but not put it at the foundation of our ethics. For example, pleasures and pains evolved to ensure survival and reproduction, i.e. to preserve our organized complexity. Of course, we acknowledge that there are many reasons to promote happiness: stress diminishes fitness for reproduction (see e.g. Moberg 1985), whereas happy people have positive emotions that allow them to broaden and build their selves, relationships and environement (e.g. Fredrickson 2004).

Instead of trying to argue for an absolute foundation, this ethical framework is provably relative. Indeed, the problem of assessing logical depth belongs to the class of non-computable problems. We would never be able to prove that the logical depth is the correct one, because a shorter value may be found in the future. However, this non-computability is not a fundamental obstacle to its use, as with Kolmogorov complexity, which is also non-computable is still largely used for concrete applications (e.g. Varré, Delahaye, and Rivals 1999; Belabbes and Richard 2008). So we can reasonably hope that similar tools will approximate logical depth (see e.g. Zenil, Delahaye, and Gaucherel 2012; Gauvrit et al. 2017).

Another issue is the measurement of the depth of an isolated object. To what do we compare its organized complexity? That is, since when do we consider the history of our object? For example, to assess the computational 
content of a human being, do we say that it starts with its birth, to its parent's birth, or should we go back to the origin of life, or even to the origin of the universe that allowed its atoms to exist? This is the temporal boundary issue that is not specific to our approach, as it also appears in holistic approaches, such as the idea of emergy (Hau and Bakshi 2004, 221a): should we take into account all the solar energy that has been used since the birth of the solar system to assess a content in solar energy of an object? If not, since when do we start?

In practice, we can't yet compute the value of any choice deterministically, to solve systematically ethical issues. Instead, a multiple-level ethics requires to solve the question: What action preserves and augments complexity at all levels? For example, deciding on whether to abort or not requires creative, mutiple levels, multiple constraints problem-solving considerations, including biological, psychological, familial, societal, religious aspects. Often, philosophers emphasize a central moral conflict: between the interests of the individual and the collective. But this need not to be limited to two levels, as we grow our circles of compassion, many more levels must be included (see Fig. 1). Again, this delicate issue regarding multiple levels is not specific to the ethics of organized complexity, but is a general problem in any complex issue, ethical or not, that involves multiple levels, aspects and stakeholders.

\section{Conclusion}

We have put forward a new foundation for ethical theories, by showing that organized complexity can be treated as an intrinsic and universal value. Such a non-anthropocentric, universal ethical foundation is much needed in our digital era. We have argued that organized complexity measures an intrinsic value: the history of non-trivial steps that have occurred to produce an object.

We put forward three imperatives of the ethics of organized complexity: to preserve, augment, and recursively promote what preserves and augments organized complexity. There are still many difficulties that lie ahead to apply those imperatives in practice. However, we saw that many such difficulties also exist in other ethical theories, and thus do not constitute a particular weakness of our approach.

The potential of such a universal ethics is great, as it can be used to develop transhumanist ethics, machine ethics, or extraterrestrial ethics. It validates many intuitive notions of good and bad, such as the value of endangered species or works of art. An original non-anthropocentric conclusion is that deep, inanimate objects have value. Few ethical theories are able to reach this conclusion. In sum, we have proposed a robust foundation for universal ethics that is promises to shed light on past, present and future ethical issues. 


\section{References}

Adriaans, Pieter. 2010. "A Critical Analysis of Floridi's Theory of Semantic Information." Knowledge, Technology \& Policy 23 (1-2): 41-56. doi:10.1007/s12130-0109097-5.

Agar, Nicholas. 2001. Life's Intrinsic Value; Science, Ethics, and Nature. New York: Columbia University Press.

Anderson, Michael, and Susan Leigh Anderson. 2011. Machine Ethics. Cambridge University Press.

Beer, Stafford. 1997. "The Culpabliss Error: A Calculus of Ethics for a Systemic World." Systems Practice 10 (4): 365-80. doi:10.1007/BF02557886.

Belabbes, Sihem, and Gilles Richard. 2008. "Spam Filtering without Text Analysis." In Global E-Security, edited by Hamid Jahankhani, Kenneth Revett, and Dominic Palmer-Brown, 144-52. Communications in Computer and Information Science 12. Springer Berlin Heidelberg. doi:10.1007/978-3-540-69403-8_18.

Bennett, C.H. 1988. "Logical Depth and Physical Complexity." In The Universal Turing Machine: A Half-Century Survey, edited by R. Herken, 227-257. Oxford University Press. bennetc/UTMX.pdf.

http://researcher.watson.ibm.com/researcher/files/us1990. "How to Define Complexity in Physics and Why." In Complexity, Entropy, and the Physics of Information, edited by Wojciech H. Zurek, 137-48. Santa Fe Institute Studies in the Sciences of Complexity, v. 8. Redwood City, Calif: AddisonWesley Pub. Co.

Bentham, J. 1907. An Introduction to the Principles of Morals and Legislation. Oxford: Clarendon Press. http://www.econlib.org/library/Bentham/bnthPML0.html.

Berlekamp, Elwyn R., John Horton Conway, and Richard K. Guy. 2001. Winning Ways for Your Mathematical Plays. 2nd ed. Natick, Mass: A.K. Peters.

Blumer, Anselm, Andrzej Ehrenfeucht, David Haussler, and Manfred K. Warmuth. 1987. “Occam's Razor." Information Processing Letters 24 (6): 377-380. http://www.cse.buffalo.edu/ hungngo/classes/2008/694/papers/occam.pdf.

Bynum, Terrell Ward. 2006. "Flourishing Ethics." Ethics and Information Technology 8 (4): 157-73. doi:10.1007/s10676-006-9107-1.

Chaisson, E. J. 2001. Cosmic Evolution: The Rise of Complexity in Nature. Harvard University Press.

Chambers, Jean. 2001. "A Cybernetic Theory of Morality and Moral Autonomy." Science and Engineering Ethics 7 (2): 177-92. doi:10.1007/s11948-001-0039-1.

Chevaleyre, Yann, Paul E. Dunne, Ulle Endriss, Jérôme Lang, Michel Lemaitre, Nicolas Maudet, Julian Padget, Steve Phelps, Juan A. Rodriguez-Aguilar, and Paulo Sousa. 2006. "Issues in Multiagent Resource Allocation." Informatica 30 (1). http://wen.ijs.si/ojs-2.4.3/index.php/informatica/article/viewFile/70/62.

Coren, Richard L. 1998. The Evolutionary Trajectory: The Growth of Information in the History and Future of Earth. The World Futures General Evolution Studies, v. 11. Amsterdam, Netherlands: Gordon and Breach Publishers.

Delahaye, Jean-Paul. 1994. Information, complexité et hasard. Paris: Hermes Science Publications.

- - - 2009. Complexité Aléatoire et Complexité Organisée. Sciences En Questions. Versailles: Quae.

Delahaye, Jean-Paul, and Clément Vidal. 2018. "Organized Complexity: Is Big History a Big Computation?" American Philosophical Association Newsletter on Philosophy and Computers 17 (2). http://arxiv.org/abs/1609.07111.

Dennett, Daniel Clement. 1995. Darwin's Dangerous Idea: Evolution and the Meanings of Life. New York: Simon \& Schuster.

Floridi, L., ed. 2003. The Blackwell Guide to the Philosophy of Computing and Information. Blackwell Publishing. 
- - 2008. "Information Ethics: Its Nature and Scope." In Information Technology and Moral Philosophy, edited by Jeroen van den Hoven and John Weckert, 40-65. Cambridge University Press.

Fredrickson, Barbara L. 2004. "The Broaden-and-Build Theory of Positive Emotions." Philosophical Transactions of the Royal Society B: Biological Sciences 359 (1449): 1367-78. doi:10.1098/rstb.2004.1512.

Freitas Jr, R. A. 1979. Xenology: An Introduction to the Scientific Study of Extraterrestrial Life, Intelligence, and Civilization. Xenology Research Institute (1979-2010). http://www.xenology.info/Xeno.htm.

Gauvrit, Nicolas, Hector Zenil, Fernando Soler-Toscano, Jean-Paul Delahaye, and Peter Brugger. 2017. "Human Behavioral Complexity Peaks at Age 25." PLOS Computational Biology 13 (4): e1005408. doi:10.1371/journal.pcbi.1005408. http://journals.plos.org/ploscompbiol/article? id=10.1371/journal.pcbi.1005408.

Hammond, Dick. 2005. Human System from Entropy to Ethics. Dick E. Hammond.

Hau, Jorge L, and Bhavik R Bakshi. 2004. "Promise and Problems of Emergy Analysis." Ecological Modelling, Through the MACROSCOPE: the legacy of H.T. Odum, 178 (1-2): 215-25. doi:10.1016/j.ecolmodel.2003.12.016.

Heylighen, F. 2007. "Why Is Open Access Development so Successful? Stigmergic Organization and the Economics of Information." In Open Source Jahrbuch 2007, edited by B. Lutterbeck, M. Baerwolff \& R. A. Gehring, 165-80. Lehmanns Media. http://pespmc1.vub.ac.be/Papers/OpenSourceStigmergy.pdf.

Heylighen, F., and J. Bernheim. 2000. "Global Progress I: Empirical Evidence for Ongoing Increase in Quality-of-Life." Journal of Happiness Studies 1 (3): 323-49. doi:10.1023/A:1010099928894.

http://pespmc1.vub.ac.be/Papers/ProgressI\&II.pdf.

Koomey, J., S. Berard, M. Sanchez, and H. Wong. 2011. "Implications of Historical Trends in the Electrical Efficiency of Computing." IEEE Annals of the History of Computing 33 (3): 46-54. doi:10.1109/MAHC.2010.28.

Korbitz, Adam. 2010. "Metalaw and SETI: Xenology, Metalaw and Thermoethics." Metalaw and SETI. December 2. http://web.archive.org/web/20110708053037/http://metalawandseti.blogspo t.com/2010/12/xenology-metalaw-and-thermoethics.html.

Li, Ming, John Tromp, and Paul Vitányi. 2003. "Sharpening Occam's Razor." Information Processing Letters 85 (5): 267-74. doi:10.1016/S0020-0190(02)00427-1.

Li, Ming, and P. M. B. Vitányi. 1992. "Inductive Reasoning and Kolmogorov Complexity." Journal of Computer and System Sciences 44 (2): 343-84. doi:10.1016/00220000(92)90026-F.

- - - 2008. An Introduction to Kolmogorov Complexity and Its Applications. Springer.

Livio, M. 2000. The Accelerating Universe. New York, Wiley \& Sons.

Maienschein, Jane, and Michael Ruse, eds. 1999. Biology and the Foundation of Ethics. Cambridge Studies in Philosophy and Biology. Cambridge, UK; New York: Cambridge University Press.

Mayfield, John. 2013. The Engine of Complexity: Evolution as Computation. Columbia University Press.

Meltzer, Michael. 2010. When Biospheres Collide: A History of NASA's Planetary Protection Programs. NASA Aeronautics and Space Administration. https://www.nasa.gov/pdf/607072main_WhenBiospheresCollide-ebook.pdf.

Moberg, Gary P. 1985. "Influence of Stress on Reproduction: Measure of Well-Being." In Animal Stress, 245-67. Springer, New York, NY. doi:10.1007/978-1-4614-75446_14.

Monod, Jacques. 1972. Chance and Necessity: An Essay on the Natural Philosophy of Modern Biology. New York: Vintage Books.

Næss, Arne. 2008. "The Shallow and the Deep, Long-range Ecology Movement. A Summary." Inquiry, August. doi:10.1080/00201747308601682. 
Odum, Howard T. 2007. Environment, Power, and Society for the Twenty-First Century: The Hierarchy of Energy. New York: Columbia University Press.

Ostwald, Wilhelm. 1912. Der Energetische Imperativ. Leipzig: Akademische verlagsgesellschaft $\mathrm{m}$. b.

http://archive.org/details/derenergetische00ostwgoog.

Quintelier, Katinka, Linda Van Speybroeck, and Johan Braeckman. 2011. "Normative Ethics Does Not Need a Foundation: It Needs More Science." Acta Biotheoretica 59 (1): 29-51. doi:10.1007/s10441-010-9096-7.

Ramezani, Sara, and Ulle Endriss. 2010. "Nash Social Welfare in Multiagent Resource Allocation." In Agent-Mediated Electronic Commerce. Designing Trading Strategies and Mechanisms for Electronic Markets, 117-31. Lecture Notes in Business Information Processing. Springer, Berlin, Heidelberg. doi:10.1007/978-3-64215117-0_9. https://pure.uva.nl/ws/files/20631648/74912_312938.pdf.

Regan, Tom. 1987. "The Case for Animal Rights." In Advances in Animal Welfare Science 1986/87, 179-89. Advances in Animal Welfare Science 1986/87. Springer, Dordrecht. doi:10.1007/978-94-009-3331-6_15.

Ruse, M. 1985. "Is Rape Wrong on Andromeda? An Introduction to Extraterrestrial Evolution, Science, and Morality." In , 43-78.

Sidgwick, Henry. 1907. The Methods of Ethics. Hackett Publishing.

Steele, Robert David. 2012. The Open-Source Everything Manifesto: Transparency, Truth, and Trust. Berkeley, Calif: Evolver Editions.

Strick, James Edgar. 2000. Sparks of Life: Darwinism and the Victorian Debates over Spontaneous Generation. Cambridge, Mass: Harvard University Press.

Ulgiati, Sergio, and Mark T. Brown. 2009. "Emergy and Ecosystem Complexity." Communications in Nonlinear Science and Numerical Simulation 14 (1): 310-21. doi:10.1016/j.cnsns.2007.05.028.

Vakoch, Douglas A., ed. 2014. Extraterrestrial Altruism: Evolution and Ethics in the Cosmos. The Frontiers Collection. Springer Berlin Heidelberg.

Varré, J. S., J. P. Delahaye, and E. Rivals. 1999. "Transformation Distances: A Family of Dissimilarity Measures Based on Movements of Segments." Bioinformatics 15 (3): 194-202. doi:10.1093/bioinformatics/15.3.194. http://bioinformatics.oxfordjournals.org/content/15/3/194.

Vidal, C. 2014. The Beginning and the End: The Meaning of Life in a Cosmological Perspective. New York: Springer. http://arxiv.org/abs/1301.1648.

- - - 2015. "A Multidimensional Impact Model for the Discovery of Extraterrestrial Life." In The Impact of Discovering Life Beyond Earth, edited by Steven J. Dick, 5575. DOI:10.1017/CB09781316272480.006. Cambridge: Cambridge University Press. http://tinyurl.com/vidal2015b.

Zenil, Hector, Jean-Paul Delahaye, and Cédric Gaucherel. 2012. "Image Characterization and Classification by Physical Complexity." Complexity 17 (3): 26-42. doi:10.1002/cplx.20388. http://arxiv.org/abs/1006.0051. 